\title{
Beiträge zur Kenntnis der Brunnenfauna im Tal des Flusses Bela Reca (Rumänien)
}

\author{
Von Constantin Motas und \\ Iosif $\mathrm{CAPUSE}^{1}$ )
}

Mit 2 Abbildungen im Text

\section{Einleitung}

Es ist bekannt, daß das Studium der Brunnenfauna gegen Ende des achtzehnten Jahrhunderts mit F.P.v.Schrank (1781) in Österreich und J. Meyer in Böhmen begann.

Aber Fortschritte machten unsere Kenntnisse über diese Tierwelt erst später. Im neunzehnten Jahrhundert zeitigten die Forschungen über die Fauna der Brunnen besonders interessante Ergebnisse. Wir erwähnen folgende Forscher: Leach (1812) meldet aus Brunnen Englands einen unterirdischen Gammariden, der nichts anderes sein kann als ein Mitglied der Gattung Niphargus; E. Pratz (1866); der die Fauna der Brunnen von St. Petersburg untersucht hat; M. Braun (1881) untersuchte die Fauna der Brunnen in Dorpat (Estland); Fr. Vejdovsky (1882) schrieb eine große Arbeit über die Brunnenfauna Prags; R. Moniez (1888) machte die Brunnentierwelt aus der Umgebung der Stadt Lille in Nordfrankreich bekannt; D. Sostaric (1888) diejenige der Brunnen Zagrebs (Kroatien); Ch. Chilton (1894) diejenige der phreatischen Gewässer Neuseelands und Australiens; A. Jaworoswky (1895) untersuchte die Brunnenfauna in Krakau und Lemberg; A. Garbini (1896) diejenige aus den phreatischen Gewässern bei Verona (Italien).

Die Fauna der artesischen Brunnen in Oued Rhir (Sahara) wurde durch G. Rolland (1894) bekanntgemacht; R. Jeannel (1937, 1950) erwähnte die aus Brunnen artesischen Typs in Texas (USA) ausgestoßenen Tierarten.

Das zwanzigste Jahrhundert zeitigte bemerkenswerte Fortschritte in der Kenntnis der Brunnenfauna durch die Untersuchungen von P. A. Chappuis (1922) über die Brunnenfauna aus der Umgebung

1) Institutul de Speologie „Emil G. Racovitza“ str. Dr. Capşa 8, Bucureşti 15 (Rumänien). 
Basels (Schweiz) und von S. Karaman (1935) über diejenige aus der Umgebung Skopjes; letzterer fand in Tr. Petkowski einen Nachfolger im Studium unterirdischer Gewässer. Zu erwähnen ist, daß S. Karaman die Methode der Grundwasserbohrungen in Jugoslawien (WardarTal) eingeführt hat, was auch Chappuis (1936, S. 389) anerkannt hat.

Vejdovsky, Chappuis und Karaman können als die Gründer eines neuen Kapitels der Hydrobiologie, die einer von uns (Motaş) als Phreatobiologie (1957, 1958a und 1958b) bezeichnet hat, betrachtet werden.

Obwohl seit 1920 durch die Niederlassung E. G. Racovitzas in unserem Lande und durch die Gründung des Institutes für Speologie in Cluj das Zentrum der speologischen Forschung in unser Land verlegt wurde, wurde die Brunnenfauna unseres Gebietes nur sporadisch untersucht.

So beschreibt R. Codreanu im Jahre 1943 eine neue Tricladenart, Dendrocoelum (Dendrocoelides) clujanum, die in einem Brunnen aus der Nähe von Cluj von Val. Puşcariu erbeutet wurde.

Nach 1944 meldeten C. Motaş und seine Schüler (J. Tanasachi und Tr. Orghidan) die Anwesenheit zahlreicher neuer Hydrachnellae in den phreatischen Gewässern verschiedener Gebiete Rumäniens, wie zum Beispiel Dacothyas sasulescui, Azugofeltria mira, Erebaxonopsis brevipes, Bogatia maxillaris (syn. Jugohydracorus acherontis) usw. C. Motaş und Tr. Orghidan (1948) erwähnten in verschiedenen phreatischen Gewässern Rumäniens 5 Arten von Cladocerae, darunter: Macrotrix laticornis var. bialatus und Alona quadrangularis O.F. Müller. C. Motaş, E. Dobreanu und C. Manolache fanden in den phreatischen Gewässern verschiedener Gebiete Rumäniens 5 phreatophile Arten von Niphargidae, darunter zwei neue Formen: Niphargus skopljensis phreaticolus und Niphargus foreli somesensis. J.Tanasachi und Tr. Orghidan (1955) beschreiben zwei neue, durch das unterirdische Leben stark beeinflußte Arten von blinden Hydracarinen: Mideopsis (Nudomideopsis) fonticola und Phreatohydracarus mosticus. D. Dancau (1962) deutet die Anwesenheit der Art Niphargus gallicus Schell. (syn. Niphargus fontanus Bate) in zahlreichen Brunnen zwischen Agigea und Tekirghiol (reg. Dobrogea) und von Baia de Fer (Reg. Oltenia) an. Von den Niphargiden erwähnt der gleiche Verfasser in den letzten Jahren die Anwesenheit in verschiedenen phreatischen Gewässern aus Rumänien der Arten: Niphargus dacicus n.sp., N.jovanovici ponoricus n.ssp., N.dobrogicus n.sp. (in litt.) usw. C. Motaş, L. Botoşaneanu und St. Negrea (1962) fanden in den Quellen des Zentralteiles des rumänischen Tales zahlreiche interessante Arten von Wirbellosen, darunter auch ein interessantes Wassermilben-Relikt, Tartarohyas romanica Husiat. (1 $\overbrace{}^{\jmath}$ und 5 qq $)$, das fast 
gleichzeitig von O. Lundblad in Schweden angegeben wurde (1962). Dies erweitert das Areal der Art wesentlich und beweist, daß die Fauna der epigeischen Gewässer dièses Landes im Eisdriftalter nicht vollkommen vernichtet werden konnte.

\section{Das untersuchte Gebiet}

Der Fluß Bela Reca ist der größte Zufluß der Cerna, die sich ihrerseits in die Donau ergießt.

Die dem Lauf der Bela Reca benachbarten Regionen sind charakterisiert durch mittlere Höhe und sind die Fortsetzung des Godeanumassivs. Die Verbindung zwischen dem Godeanugebirgsstock und den Gebirgszügen des Banates wird durch eine Bergregion hergestellt, die dem Becken der Cerna angehört und die das Cerna- und die Mehedintigebirge mit dem zwischen ihnen tief eingeschnittenen Tal der Cerna umfaßt.

Der Unterlauf der Cerna trennt das Hochplateau und die Gebirge von Mehedinti von den Almaşbergen, die nördlich den Durchbruch der Donau begleiten. Charakteristisch für diesen Gebirgszug ist der 10001200 m hohe Hauptkamm, von dem eine Reihe von Höhenzügen abzweigen, die nach und nach bis $400-500 \mathrm{~m}$ in der Nähe des Donaudurchbruchs abfallen. An ihrer geologischen Zusammensetzung sind besonders kristallinischer Schiefer, Eruptivformationen und in untergeordnetem Maße sedimentäre Formen beteiligt.

Die von uns untersuchten Brunnen liegen im Bela-Reca-Tal zwischen Mehadia und dem Bahnhof Baile Herculane (Kreis Orşova, Region Banat). Die wasserführende Schicht liegt in einer alluvialen

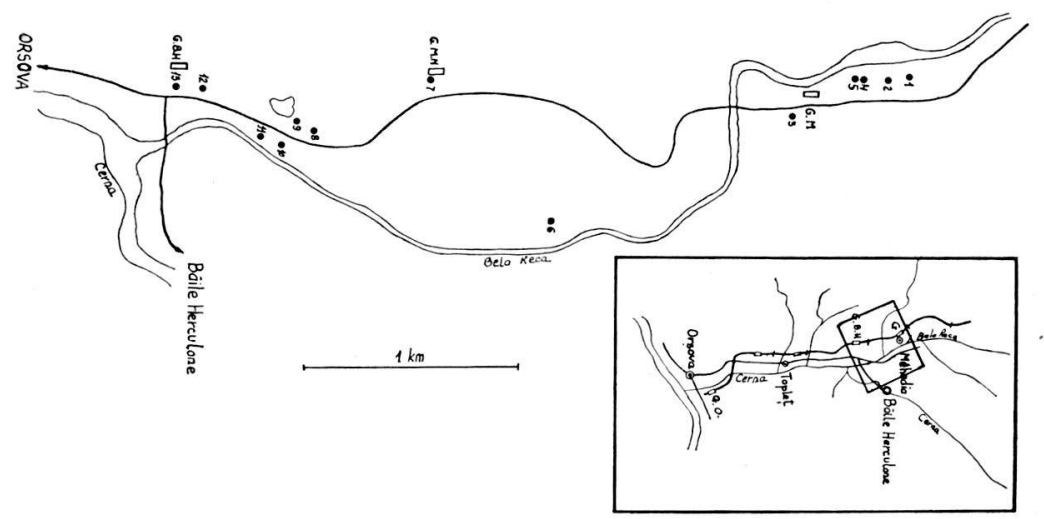

Abb. 1. Lageskizze der untersuchten Brunnen. 
Terrasse von $168 \mathrm{~m}$ Seehöhe. Das von uns untersuchte Tal wird von 300-700 m hohen Bergzügen begleitet. Die Mehrzahl der Berge sind mit aus verschiedenen Holzarten bestehenden Wäldern bedeckt, nur wenige sind baumfrei.

Die dem Bela-Reca-Tal benachbarten Berge bestehen aus Eruptivgesteinen, Schiefern, Konglomeraten und sehr wenig Kalk, der in der Nähe des Zusammenflusses des Bela Reca und Cerna einen größeren Anteil an der Gesteinsformation hat.

Die Alluvionalterrasse weist verschiedene Elemente, die dem Zerfall der Gesteine zuzuschreiben sind, auf. Es herrschen Quarzkonglomerate und -sand vor.

In dieser Gegend haben wir folgende 13 Brunnen wiederholt und zu verschiedenen Jahreszeiten untersucht:

1. Brunnen Trapcea Iosif,

2. Brunnen Ionescu Ion,

3. Brunnen Guleran Tiberiu,

4. Brunnen Capuşe Elisabeta I,

5. Brunnen Capuşe Elisabeta II,

6. Brunnen Colojoara Aurel,

7. Brunnen des Bahnhofs Mehadia Noua,

8. Brunnen I beim Bahnwärterhaus (1 km oberhalb des Bahnhofs Baile Herculane),

9. Brunnen II bei demselben Bahnwärterhaus,

10. Brunnen Capuşe Elisabeta III,

11. Brunnen Trapcea Ionita,

12. Brunnen des Bahnwärterhauses beim Bahnhof Baile Herculane,

13. Brunnen des Bahnhofs Baile Herculane.

All diese Brunnen sind mit Holzbrettern eingefaßt und mit Steinen ausgefüttert. Auf dem sandigen Grund der Brunnen haben wir hineingefallene organische Substanzen feststellen können, bestehend aus Laub und zufällig im Brunnen ertrunkenem Kleingetier.

Zwecks leichterer Orientierung geben wir hier eine Skizze der untersuchten Gegend.

\section{Fangmethode}

Für den Fang der Tiere wurden mehrere Methoden angewandt. Der größte Teil der Fänge geschah durch Durchziehen des Keschers in verschiedenen Tiefen des Brunnens. Ein Teil des erbeuteten Materials wurde an Ort und Stelle, der andere Teil im Laboratorium mit der Binokularlupe gesichtet. 
Es wurden auch größere Tiere (Isopoden, Amphipoden) an der Oberfläche untergetauchten Holzes und im Wasser liegender Steine gesammelt.

Außer diesen beiden Methoden haben wir auch das Senknetz benützt, das mit Wurst, Schafkäse und etwas angefaultem Fleisch beködert war.

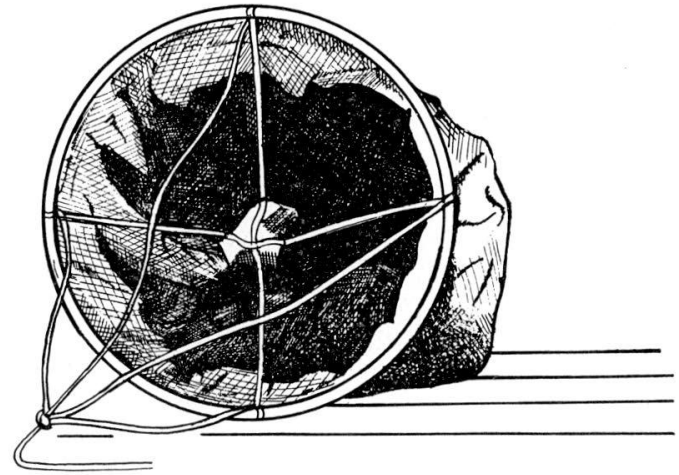

Abb. 2. Senknetz für den Fang der Brunnenfauna.

\section{Physische und chemische Beschaffenheit des Wassers}

Die Sommertemperatur des Wassers in den Brunnen schwankt zwischen $+12^{\circ}$ und $+14^{\circ} \mathrm{C}$. Die Temperatur der meisten Brunnen beträgt $+12^{\circ} \mathrm{C}$. Ein einziger Brunnen $\left(\mathrm{F}_{9}\right)$ wies eine Temperatur von $+16^{\circ} \mathrm{C}$ auf, was dem Umstande zuzuschreiben ist, daß sein Wasser aus dem benachbarten Sumpf stammt.

Temperaturunterschiede von $+1^{\circ}-+2^{\circ} \mathrm{C}$ zwischen den einzelnen Brunnen sind ihrer verschiedenen Tiefe zuzuschreiben. Das der Oberfläche näher liegende Wasser erwärmt sich mehr als das der tieferen Brunnen.

Während des Winters $(1956,1957,1958)$ betrug die Wassertemperatur $+11^{\circ}-+12^{\circ} \mathrm{C}$. Der Unterschied zwischen der Sommer- und Wintertemperatur von $2^{\circ}-3^{\circ} \mathrm{C}$ weist auf die Stenothermie der phreatischen Gewässer hin.

Das Wasser der Brunnen ist farblos und durchsichtig und gestattet die Bewegungen der darin befindlichen Isopoden und Amphipoden zu beobachten. Nur im Frühjahr, wenn der Brunnenspiegel steigt, ist der Boden nicht mehr sichtbar.

Das Wasser der Brunnen ist geruchlos, nur die nichtgeputzten Brunnen weisen beim Schütteln des Wassers einen Fäulnisgeruch auf, 
welcher der Zersetzung des Laubes und der Leichen von Tieren, die in den Brunnen gefallen sind, zuzuschreiben ist.

Die untersuchten Brunnen liegen 30-400 m vom Bett des Flusses Bela Reca entfernt.

Über die chemische Zusammensetzung des Wassers gibt die aufgeführte Tab. 1, die die chemische Analyse von 2 in einer Entfernung von $4 \mathrm{~km}$ voneinanderliegenden Brunnen wiedergibt, Aufschluß:

Tabelle 1

\begin{tabular}{|c|c|c|c|}
\hline & & Quelle $\mathrm{F}_{4}$ & Quelle $\mathrm{F}_{10}$ \\
\hline $\mathrm{pH}$ & & 7,00 & 6,90 \\
\hline Alkalinität ccm $\mathrm{HCl} \mathrm{n/l}$ & & 7,32 & 5,28 \\
\hline $\begin{array}{l}\text { Vorübergehende Härte, deutsc } \\
\text { Härtegrade }\end{array}$ & & 20,49 & 14,78 \\
\hline Verbrauch von $\mathrm{KMnO}_{4}$ & $\mathrm{mg} / \mathrm{l}$ & 18,18 & 21,86 \\
\hline $\begin{array}{l}\text { Fester Rückstand } 105^{\circ} \\
\text { nach Schütteln des Wassers } \\
\text { in dekantiertem Wasser }\end{array}$ & $\begin{array}{l}\mathrm{mg} / \mathrm{l} \\
\mathrm{mg} / \mathrm{l}\end{array}$ & $\begin{array}{l}1430,00 \\
1340,00\end{array}$ & $\begin{array}{l}368,00 \\
290,00\end{array}$ \\
\hline Schwebstoffe & $\mathrm{mg} / \mathrm{l}$ & 90,00 & 78,00 \\
\hline Nitrate $\mathrm{N}_{2} \mathrm{O}_{5}$ & $\mathrm{mg} / \mathrm{l}$ & 6,60 & Spuren \\
\hline Phosphate $\mathrm{P}_{2} \mathrm{O}_{5}$ & $\mathrm{mg} / \mathrm{l}$ & - & - \\
\hline $\mathrm{NaCl}$ & $\mathrm{mg} / \mathrm{l}$ & 187,00 & 26,30 \\
\hline $\mathrm{Ca}$ & $\mathrm{mg} / \mathrm{l}$ & 281,30 & 73,30 \\
\hline Eisen (qualitativ) & & Spuren & Spuren \\
\hline
\end{tabular}

Aus der chemischen Analyse der beiden Proben ergibt sich, daß das Wasser neutral reagiert. Bemerkenswert ist die Menge an Ca im Wasser des Brunnens $\mathrm{F}_{4}$ und als Folge hiervon die große Härte dieses Wassers.

In den Brunnen ist organische Substanz in genügender Menge vorhanden. Sie stammt von Tierleichen und pflanzlichen Abfällen, die von der Oberfläche her in die Brunnen gelangten. In einigen Brunnen $\left(F_{11}\right)$ ist sie in größerer Menge vorhanden als in anderen $\left(F_{5}\right)$.

\section{SYSTEMATISCHER TEIL}

\section{Tricladida}

Wir fanden ein einziges, vollkommen pigmentfreies Exemplar von einer noch unbestimmten Planarienart (Prof. R. Codreanu). Obwohl wir $F_{11}$ sehr oft $\mathrm{zu}$ allen Zeiten des Jahres untersuchten, ist es uns 
nicht gelungen, weitere Exemplare von Planarien zu erbeuten. Da das gefundene Material nicht genügte, konnte eine Bestimmung nicht vorgenommen werden.

\section{Archioligochaeta (det. N. Toniuc)}

Die Gattung Rhyacodrilus Bretscher 1901 hat gegabelte Chaeten, in Form einer zweizinkigen Gabel. Ein echter Penis fehlt. Die Zahl der Segmente beträgt 40-65. Die Arten dieser Gattung sind in Deutschland, Dänemark, Böhmen und der Schweiz verbreitet. Unter dem von uns erbeuteten Material wurden folgende Arten in allen untersuchten Brunnen identifiziert:

1. Rhyacodrilus coccineus (Vejdovsky) lebt in klaren Bächen und fließenden Gewässern.

2. Rhyacodrilus palustris (Dietlevsen) in denselben Biotopen wie die vorhergehende Art.

3. Rhyacodrilus falciformis (Bretscher) findet sich gewöhnlich im Sand der Quellen und Teiche.

\section{Gastropoda (det. A. V. Grossu)}

1. Cochlicopa lubrica (Mull.).

2. Vallonia pulchella (Muoll.).

Beide Arten wurden in den Brunnen $\mathrm{F}_{10}$ und $\mathrm{F}_{11}$ gefunden. Sie gehören der Ordnung Stylommatophora (Landlungenatmer) an. Sie bevorzugen feuchte Standorte und werden gewöhnlich auf Moos und Steinen in unmittelbarer Nähe des Wassers gefunden. In das Wasser der Brunnen sind sie zufällig hineingeraten.

\section{Cladocera (det. St. Negrea)}

1. Daphnia pulex de Geer. Zahlreiche Weibchen mit parthenogenetischen Eiern, wenige mit Ephippien; desgleichen wurden auch jugendliche Exemplare in den Brunnen $\mathrm{F}_{1}, \mathrm{~F}_{2}, \mathrm{~F}_{3}, \mathrm{~F}_{4}, \mathrm{~F}_{6}, \mathrm{~F}_{7}, \mathrm{~F}_{9}, \mathrm{~F}_{10}$ und $\mathrm{F}_{11}$ gefunden. Die erbeuteten Tiere hatten Eier und zahlreiche Embryos; ein Beweis, daß sich die Art in Brunnen normal entwickelt. Gewöhnlich findet sich diese Art in wenig tiefen Teichen, aber auch in großen Landseen, in der Litoralzone. D. pulex de Greer ist eine kosmopolitische Art, die unter allen Klima- und Höhenverhältnissen angetroffen wird. Diese Art wurde auch durch R. Moniez (1888-1889) in den unterirdischen Gewässern bei Lille (Frankreich) wie auch von A. Garbini im Grundwasser (1896) bei Verona angetroffen. 
2. Simocephalus exspinosus Koch. Wir fanden Weibchen mit Eiern und Jungtiere in den Brunnen $\mathrm{F}_{8}$ und $\mathrm{F}_{12}$. Die Art lebt besonders in kleinen Gewässern und ist in der gemäßigten Zone allgemein verbreitet. Es ist die häufigste Art der Gattung Simocephalus.

3. Ceriodaphnia reticulata G. O. Sars. Wir fingen Weibchen mit Eiern in den Brunnen $\mathrm{F}_{8}$ und $\mathrm{F}_{10}$. Es ist eine für gut gedüngte künstliche Teiche charakteristische Art. Sie ist häufig in kleinen und am Ufer größerer Wasserbecken in den Zonen mit Pflanzenwuchs. Sie wurde auch von R. Moniez (1888-1889) aus Brunnen von Lille (Frankreich) gemeldet.

4. Ceriodaphnia laticaudata P. E. Muller. Wir sammelten Weibchen mit Eiern und Jungen und einzelne Ephippien in $F_{2}, F_{3}$ und $F_{11}$. Es ist die am weitesten verbreitete Art und vielleicht der am meisten verbreitete Cladocere. Ch.sphaericus ist in allen Gewässern zu finden, am Ufer, auf dem Grunde und im Plankton, da sie gegen pH-Schwankungen unempfindlich ist. Diese Art wurde auch von H. Spandl (1926) in einer Höhle in Mähren und von C. Motaş und Tr. Orghidan (1948) im Tal des Flusses Bogata gefunden. Außer dieser Art fanden die beiden letztgenannten Forscher im Grundwasser des Flusses Bogata folgende Arten: Macrothrix laticornis var. bialatus Motaş \& Orghidan, Alona quadrangularis O.F. Müller, Iliocryptus sordidus Lievin und Leydigia leydigi Schoedler.

\section{Ostracoda (det. S. Beldescu)}

Candona eremita (Vejdovsky). Sie wurde in großer Menge in den Brunnen $\mathrm{F}_{5}, \mathrm{~F}_{6}, \mathrm{~F}_{7}, \mathrm{~F}_{8}, \mathrm{~F}_{10}, \mathrm{~F}_{11}$ und $\mathrm{F}_{13}$ gefunden. Sie ist blind und nicht pigmentiert und findet sich ausschließlich im phreatischen Biotop. Sie ist ein Bestandteil dessen, was einer von uns (Motaş, 1958) Phreaton (= Biozenose des phreatischen Wassermediums) genannt hat.

Racovitza und Jeannel fanden sie wieder in einer Höhle (Podpec bei Gottschen) in Kärnten (Biospeol. Nr. 634, 1920) und Chappuis (1922) in einem Brunnen aus Basel.

\section{Cyclopida (det. A. Damian)}

1. Acanthocyclops bicuspidatus (Claus). Wir fanden zahlreiche Exemplare in den Brunnen $\mathrm{F}_{2}, \mathrm{~F}_{4}$ und $\mathrm{F}_{11}$. Diese Art lebt in großen Gewässern wie auch in kleinen, nur zeitweilig auftretenden Tümpeln, in Morästen, am Ufer von Teichen, in fließenden Gewässern und Quellen vom limnokrenen Typus. In Landseen geht sie nicht tiefer als $10 \mathrm{~m}$. A. bicuspidatus (Claus) wurde in Gewässern in Höhlen und in Brunnen gefunden. Es ist eine sehr häufige Art und findet sich auch in stark kochsalzhaltigen Gewässern. 
2. Acanthocyclops prasinus (Jurine) fanden wir in viel kleinerer Menge in den Brunnen $\mathrm{F}_{6}$ und $\mathrm{F}_{11}$.

3. Trospsocyclops prasinus (Fischer). Wie die obige Art ist sie viel seltener und wurde nur in $\mathrm{F}_{10}$ gefunden.

\section{I s op oda (det. R. \& M. Codreanu)}

1. Asellus (Coxasellus) elegans R. \& M. Codreanu, n.sp. aus der Gruppe $A .(C)$. coxalis (Dollfus) Raco.

$A$. $(C$.) coxalis ist aus Syrien bekannt. Kürzlich wurde sie von S. Karaman (1950) in Jugoslawien gefunden; auch sind verschiedene andere Unterarten in der Balkanhalbinsel und in Italien bekannt. $A$. (C.) coxalis wahrt gefiederte Haare auf den Anhängen, die bei den epigäischen Arten sehr zahlreich sind.

A. (C.) elegans n. sp. ist $7 \mathrm{~mm}$ lang, vollkommen pigmentlos, von zierlichem Äußeren. Es ist eine außerordentlich widerstand sfähige Art, die nach Fixierung alle ihre Anhänge wahrt; sie kann in Kristallisierschalen mehrere Wochen überleben.

Die Antenne II ist sehr lang, der Uropod kann bei ausgewachsenen Männchen die Länge des Pleotelsons überschreiten; desgleichen sind die Pereiopoden 5-7 auffallend lang. Pleopod 2 beim Männchen mit dem Exopodit etwas länger als der Endopodit; letzterer ist, wie bei allen Isopoden, zum Kopulationsfortsatz umgewandelt, seine distale Öffnung ist eng spiralförmig; er besitzt eine hervortretende basale Apophyse.

Im Rahmen der Reihe A. (C.) coxalis und der Gruppe A. anophtalmus Karaman, nähert sich diese Art sehr stark $A$. montenegrinus und $A$. hercegovinensis Karaman.

2. Asellus (Coxasellus) danubialis R. \& M. Codreanu n.sp. aus der Gruppe A. (C.) coxalis (Dollfus 1892) Raco. 1919.

Sie lebt in der Mehrzahl der Brunnen zusammen mit der vorher genannten Art, wobei sie sich durch einen den meisten Aselliden viel mehr angenäherten Habitus und kleinere Maße, bis zu 5,5 mm unterscheidet. Desgleichen ist auch ihr Stoffwechsel verschieden, indem sich bei ihr Eisenhydrat auf der Oberfläche der Oberhaut absetzt, besonders dorsal bei den im September 1957 gesammelten Exemplaren aus dem Brunnen $\mathrm{F}_{\mathbf{1 0}}$, in welchen sich eiserne Gegenstände befanden. Die Eisenablagerungen waren so massenhaft, daß das Tier auf den ersten Blick einem pigmentierten Aselliden glich. Nur die aufmerksame Beobachtung durch das Mikroskop und die Reaktion mit Ferrocyankalium in Lösung mit Normalsalzsäure brachte das Eisen zum Vorschein. Ihre Mitbewohner der Art A. (C.) elegans hatten das Tegument voll- 
kommen rein, ohne jede Ablagerungen von $A$. (C.) danubialis abgestoßen und blieben nur auf der abgelegten Haut sichtbar. Hierbei können Exemplare angetroffen werden, die zur Hälfte mosaikartig oder in anderen Kombinationen pigmentiert sind.

Diese Art unterscheidet sich durch die morphologischen Charaktere aller Körperanhänge und besonders der Pleopoden 1-5, während der Pleopod 2 des Männchens ein äußerst charakteristisch stark gekrümmtes Endopodit aufweist.

In den Brunnen der Insel Ada-Kaleh fanden wir den $A$. (C.) danubialis zusammen mit dem Asellus aquaticus L., Nyphargus stygius und Synurella sp.

A. (C.) danubialis ist viel weiter verbreitet, denn sie wurde auch in einem Brunnen der Gemeinde Cîrlița (Dobrogea) auf Grund von im Juni 1957 durch Tr. Orghidan gesammeltem Material identifiziert.

Die extreme Lage dieser beiden Fundorte läßt das Vorhandensein dieser Art längs des ganzen Laufs der Donau als möglich erscheinen, was den Namen rechtfertigt, dem ihr die Autoren gaben.

3. Asellus aquaticus (L.) emend. Raco. 1919. Wir fingen viele Exemplare im Brunnen $\mathrm{F}_{9}$. Wahrscheinlich stammen sie aus dem dem Brunnen benachbarten Sumpf. Diese Annahme gründet sich auf die Tatsache, daß die Art in allen anderen 12 Brunnen fehlt und dort durch die vorher erwähnten Arten ersetzt wird, aber im Sumpf in der Nähe der Brunnen Nr. 8 und 9 auftritt.

Es handelt sich um eine sehr häufige Art nördlicher Herkunft. Sie ist pigmentiert, besitzt Sehwerkzeuge und war in der Nach-Eiszeit in ruhigen Oberflächengewässern sehr verbreitet. Das Eiszeitklima hat den Weiterbestand der alten Asellus-Formen aus der epigäischen europäischen Fauna nur in den extremen Regionen der drei südlichen Halbinseln Europas gestattet, so daß gegenwärtig die vorherrschende Form Asellus aquaticus ist; ein Einwanderer aus jüngster Zeit, wie ihn Racovitza in seiner bekannten Arbeit aus dem Jahre 1919 umgrenzt hat.

4. Hyloniscus riparius (C. L. Koch) (det. I. Tabacaru). Wir erbeuteten diese Art im Brunnen $\mathrm{F}_{10}$.

\section{Amphipoda}

1. Niphargopsis trispinosus Dancau \& Capuşe. Niphargopsis ist für die Amphipodenfauna unseres Landes eine neue Gattung und weist bei uns eine für die Wissenschaft neue, kürzlich (1959) unter obigem Namen beschriebene Art auf. 
N.trispinosus ist nicht pigmentiert, augenlos. Die gesammelten Exemplare sind 2,6-9,2 mm lang. Die Maxille I weist auf dem Innenlappen apikal 1-2 Haare auf. Der äußere Lappen, von viereckiger Form und stark verbreitert, ist distal mit 2-3 geraden pektinierten oder einfachen Stacheln besetzt, denen 10-11 pektinierte Stacheln von Schaufelform folgen, weiter 15 kleinere, mit zahlreichen kürzeren Haaren besetzte Dornen. Subterminal noch drei kräftige, leicht gebogene und an der Spitze scharfe Dornen. Die Art wurde in den Brunnen $\mathrm{F}_{2}, \mathrm{~F}_{5}, \mathrm{~F}_{6}, \mathrm{~F}_{10}$ und $\mathrm{F}_{11}$ gesammelt.

2. Niphargus jovanovici bajuvaricus Schell. Eine für die rumänische Fauna neue Unterart.

Körperlänge 6-7 mm. Die Gnathopoden der Unterart sind denen des Arttypus ähnlich. Der Telson ist merklich länger als breit, dreiviertel gespalten, jeder Lappen trägt 2-3 pektinierte Dornen und Haare. Die Enddornen des Uropods 3 sind kürzer als bei den anderen Unterarten.

Diese Unterart ist aus dem oberen Rheintal und den Brunnen aus der Umgebung Freiburgs bekannt. Wir fanden sie in den Brunnen $\mathrm{F}_{\mathbf{1}}$, $\mathrm{F}_{2}, \mathrm{~F}_{3}, \mathrm{~F}_{4}, \mathrm{~F}_{5}, \mathrm{~F}_{6}, \mathrm{~F}_{8}, \mathrm{~F}_{10}, \mathrm{~F}_{11}$ und $\mathrm{F}_{13}$ zusammen mit Niphargopsis trispinosus Dancau \& Capuşe.

\section{Halacaridae (det. J.Tanasachi)}

Lolohalacarus weberi (Romijn \&Viets). Ein einziges Exemplar wurde im Brunnen $\mathrm{F}_{\mathbf{1 0}}$ erbeutet.

\section{Collembola (det. M. Ionescu)}

Tomocerus sp. Einige Exemplare wurden in den Brunnen $\mathrm{F}_{\mathbf{6}}$ und $\mathrm{F}_{\mathbf{9}}$ gefunden.

\section{Trichoptera}

Drei Gehäuse von Limnophilinae in $\mathrm{F}_{6}$.

\section{Coleoptera (det. A. Decu)}

1. Helodes minuta. Gefunden wurden erwachsene Exemplare, Larven und Exuvien in $\mathrm{F}_{10}$ und $\mathrm{F}_{11}$.

2. Stenelmis sp. Ein Exemplar im Übergangsstadium zur Nymphe in $\mathrm{F}_{11}$.

Diese Coleopterenformen finden sich in Quellen und fließenden Gewässern. 


\section{Diptera}

1. Culex pipiens und 2. Culex baraulus.

Zahlreiche Larven und Nymphen beider Arten wurden in allen untersuchten Brunnen gefunden.

3. Corethra (Chaoborus) sp. Wir fanden zahlreiche Larven in $\mathrm{F}_{9}$.

Tendipetidae (det. Dr. N. Botnariuc)

4. Ablabesmia gr. tetresticta (Kieff.).

5. Tendipes forma larvalis plumosus (L).

6. Tendipes forma larvalis thummi (Kieff.).

7. Procladius sp. Puppen.

Diese 4 Formen von Tendipetidae wurden in allen Brunnen zusammenlebend vorgefunden. Ferner fanden wir noch eine Form von Dipteren, die mangels erwachsener Exemplare nicht bestimmt werden konnte. Die Nymphen dieser Form weisen gegenüber allen anderen bekannten Arten außergewöhnliche Merkmale auf. Es ist möglich, daß es sich um eine ganz neue Form handelt.

Aus der Aufzählung der in den untersuchten Brunnen gefundenen Tiergruppen ergibt sich ein Unterschied zwischen den verschiedenen Brunnen, was aus der folgenden Tab. 2 mit mehr Klarheit ersichtlich ist:

Tabelle 2

\begin{tabular}{|c|c|c|c|c|c|c|c|c|c|c|c|c|c|}
\hline $\begin{array}{l}\text { Brunnen } \\
\text { Tiergruppen }\end{array}$ & $F_{1}$ & $\mathrm{~F}_{2}$ & $\mathrm{~F}_{3}$ & $\mathrm{~F}_{4}$ & $\mathrm{~F}_{5}$ & $\mathrm{~F}_{6}$ & $\mathrm{~F}_{7}$ & $\mathrm{~F}_{8}$ & $\mathrm{~F}_{9}$ & $\mathrm{~F}_{10}$ & $\mathrm{~F}_{11}$ & $\mathrm{~F}_{12}$ & $\mathrm{~F}_{13}$ \\
\hline $\begin{array}{l}\text { Tricladida } \\
\text { Archioligochaeta } \\
\text { Gastropoda }\end{array}$ & + & + & + & + & + & + & + & + & + & + & $\begin{array}{l}+ \\
+ \\
+\end{array}$ & + & + \\
\hline $\begin{array}{l}\text { Cladocera } \\
\text { Ostracoda } \\
\text { Cyclopoida }\end{array}$ & + & $\begin{array}{l}+ \\
+\end{array}$ & + & $\begin{array}{l}+ \\
+\end{array}$ & $\begin{array}{l}+ \\
+\end{array}$ & $\begin{array}{l}+ \\
+ \\
+\end{array}$ & $\begin{array}{l}+ \\
+\end{array}$ & $\begin{array}{l}+ \\
+\end{array}$ & + & $\begin{array}{l}+ \\
+ \\
+\end{array}$ & $\begin{array}{l}+ \\
+ \\
+\end{array}$ & + & $\begin{array}{l}+ \\
+\end{array}$ \\
\hline Isopoda & + & + & + & + & + & + & + & + & + & + & + & + & + \\
\hline $\begin{array}{l}\text { Amphipoda } \\
\text { Halacaridae } \\
\text { Collembola } \\
\text { Coleoptera }\end{array}$ & + & + & + & + & + & + & & + & + & $\begin{array}{l}+ \\
+ \\
+\end{array}$ & $\begin{array}{l}+ \\
+\end{array}$ & & + \\
\hline $\begin{array}{l}\text { Trichoptera } \\
\text { Diptera }\end{array}$ & + & + & + & + & + & $\begin{array}{l}+ \\
+\end{array}$ & + & + & + & + & + & + & + \\
\hline
\end{tabular}


Aus der Aufzählung der Arten in der Tab. 2 kann festgestellt werden, daß $\mathrm{F}_{10}$ und $\mathrm{F}_{11}$ die Brunnen mit den meisten Gruppen von Tieren sind, während $F_{8}$ die wenigsten Gruppen enthält. Ferner muß erwähnt werden, daß $\mathrm{F}_{\mathbf{1 1}}$ außer der größten $\mathrm{Zahl}$ von Arten auch die größte Bevölkerungsdichte aufweist.

Bis jetzt wurden in den Brunnen des Bela-Reca-Tales bei Mehadia insgesamt 34 Arten, denen 12 Tiergruppen angehören, gefunden.

Wir erwähnen, daß in den Bohrungen, die in den alluvialen Ablagerungen des Bela-Reca-Tales ausgeführt wurden, keine Fauna gefunden wurde. Bemerkenswert ist, daß einer von uns (Motaş) zusammen mit seinen Mitarbeitern Tr. Orghidan und N. Botnariuc eine Reihe von ebenfalls ergebnislosen Bohrungen gegen Ende Oktober $1946 \mathrm{im}$ Tal dieses Flusses vornahm. Dagegen ergaben die zur gleichen Zeit ausgeführten Bohrungen im Cerna-Tal einige interessante Arten von Hydracarinen, darunter Hungarohydracarus subterranus Szalay 1943 wie auch eine neue Art einer neuen Untergattung, Axonopsis (Paraxonopsis) vietsi Motaş \& Tanasachi 1947, die von E. Angelier (1953) in den Pyrenäen wiedergefunden wurde.

Unter dem Gesichtspunkt der Anpassung an das phreatische Medium enthalten die in den untersuchten Brunnen gefundenen Arten 2 ökologische Gruppen von Elementen: 1. phreatobionte und 2. phreatoxene.

Wir glauben, daß als phreatobiont folgende Arten betrachtet werden können:

1. Candona eremita (Vejdovsky),

2. Asellus (Proasellus) elegans n. sp. R.\& M. Codreanu,

3. Asellus (Proasellus) danubialis n. sp. R.\& M. Codreanu,

4. Niphargus jovanovici bajuvaricus Schell.,

5. Niphargopsis trispinosus Dancau \& Capuşe.

Unter diesen phreatobionten Elementen sind 2 und 3 neu für die Wissenschaft, 4 neu für die Fauna Rumäniens.

Die Gattung Niphargopsis enthält nur 2 Arten, eine N.caspary (Pratz) ist aus dem Westen Europas bekannt. Die zweite, N.trispinosus Dancau \& Capuşe, kann bis zur Stunde in der untersuchten Gegend als endemisch betrachtet werden. Das gleiche gilt für die Isopodenart A. (P.) elegans R. \& M. Codreanu.

Die übrigen 27 Arten, das heißt etwa 85\%, gehören zur Kategorie der Phreatoxenen, von denen die meisten Arten von großer Verbreitung sind, die in verschiedenen Typen von epigäischen Gewässern oder in anderen Biotopen leben. 


\section{Schlußfolgerungen und Diskussion der Ergebnisse}

Die 13 untersuchten Brunnen gestatten noch nicht, endgültige Schlußfolgerungen über die Zusammensetzung der Fauna dieses Biotops zu ziehen.

Die Fauna der Brunnen von Mehadia besteht aus 34 Formen, worunter 1 Triclade (nicht identifiziert), 3 Oligochaeten, 2 Gasteropoden, 5 Cladoceren, 1 Ostracode, 3 Copepoden, 4 Isopoden, 2 Amphipoden, 1 Halacaride, 1 Colembole (nicht identifiziert), 1 Trichoptere (nicht identifiziert), 2 Coleopteren, 8 Dipteren.

Zu bemerken ist, daß 15\% der Arten zur Gruppe der Phreatobionten, $85 \%$, also die große Mehrzahl, zur Gruppe der Phreatoxenen gehört (darunter einige Erdbewohner).

Wenn man in Betracht zieht, daß Vejdovsky (1882) insgesamt 104 Arten, davon 70 Protozoen, in den Brunnen von Prag, Moniez (1888-1889) 88 Arten in den Brunnen aus der Umgebung der Stadt Lille und in Norddepartement Frankreichs und Chappuis (1922) 89 Arten in den Brunnen um die Stadt Basel (Schweiz) gesammelt hat, ist das Ergebnis der Untersuchung der Brunnen um Mehadia zufriedenstellend. Dies um so mehr, als Chappuis (op. cit.) insgesamt 120 Brunnen untersucht hat, davon 79 bis in die Grundwasserschicht gegrabene Brunnen (Sodbrunnen) und 41 Brunnenhäuser, aus denen Brunnenstuben durch Rohrleitungen versorgt werden, während sich unsere Untersuchungen nur auf 13 Brunnen erstreckten.

Was wir hervorheben müssen, ist die Tatsache, daß sowohl Vejdovsky als auch Moniez und Chappuis in den untersuchten Brunnen einen viel größeren Anteil an epigäischen als an eigentlichen unterirdischen Formen fanden; eine Tatsache, die auch wir festgestellt haben. Nach Chappuis enthalten nur 2\% der Brunnen aus der Umgebung von Basel eine ausgesprochene phreatische Fauna.

In dieser Hinsicht haben sich die phreatischen Gewässer von Skopje (Jugoslawien) als viel interessanter erwiesen, die 1930-1931 durch den leider verschiedenen S. Karaman (1889-1959) untersucht wurden. Er fand dort neue Familien von Isopoden (Microparasellidae) ${ }^{2}$ ), 2 Vertreter einer neuen Unterordnung von Amphipoden, Ingolfielliden ${ }^{3}$ ), und die ersten neuen Gattungen und Arten von durch K. Viets in einer Reihe von Mitteilungen (1931-1935) bestimmten Wassermilben.

$\left.{ }^{2}\right)$ E. Serban (in litt.) erwähnt 3 neue Arten von Microcharon in verschiedenen phreatischen Gewässern aus Rumänien.

3) Ingolfiella acherontis und I. petkosskii. Die letztere war auch in Bulgarien gefunden von L. Zvetkov (1964). 
In dieser Hinsicht, das heißt in Hinsicht auf die Fauna der Hydracarinen, wurden in Brunnen Japans eine Reihe von neuen, durch Uchida und Imamura untersuchte Gattungen entdeckt.

Im übrigen bestehen zwischen einer Grundwasserbohrung und einem Brunnen folgende Unterschiede: Jener ist eine vorübergehende Arbeit, die keinen wirtschaftlich-praktischen Zweck verfolgt; letzterer ist ein dauerndes Objekt, er dient zur Versorgung der häuslichen Wirtschaft mit Trinkwasser. Eine Grundwasserbohrung wird oft in einigen Minuten ausgeführt; ein Brunnen ist ein Bau, der Tage, sehr oft Wochen zu seiner Herstellung benötigt.

Der Brunnen ist kein besonderes Biotop, er gestattet nur einen Einblick in das phreatische Medium. Ein Beweis hierfür liegt darin, daß in den Brunnen der japanischen Inseln die Gattungen Erebaxonopsis Mot. \& Tan. 1948 gefunden wurden, die sich bei uns in dem die alluviale Anschwemmungen der Flußtäler durchtränkenden Wasser finden. Vertreter der vorgenannten Gattungen finden sich im interstitiellen Wasser der alluvialen Ablagerungen der Flußtäler, während sie in Japan in Brunnen gefunden wurden. Ferner wurde Tartarothyas fonticola Mot.\& Tan. bei uns sowohl in einem Brunnen (1 juv. ㅇ) als auch in Interstitielwasser (2 imagos) gefunden.

\section{ZUSAMMENFASSUNG}

Nach einer Einleitung, die hauptsächlich eine kurze Geschichte der Erforschung der Brunnenfauna umfaßt, folgt die Beschreibung des untersuchten Gebietes, in dem wir 13 Brunnen erforscht haben. Weiter stellen die Verfasser die Arbeitsmethode und die physische und chemische Beschaffenheit des Wassers (Temperatur, pH, Härte, Verbrauch von $\mathrm{KMnO}_{4}$, fester Rückstand, Schwebstoffe, $\mathrm{N}_{2} \mathrm{O}_{5}, \mathrm{P}_{2} \mathrm{O}_{5}, \mathrm{NaCl}, \mathrm{Ca}$ ) dar.

Die Fauna der Brunnen von Mehadia (siehe den ,systematischen Teil") besteht aus 34 Formen: 1 Triclade, 3 Oligochaeten, 2 Gasteropoden, 5 Cladoceren, 1 Ostracoden, 3 Copepoden, 4 Isopoden, 2 Amphipoden, 1 Halacaride, 1 Colembole, 1 Trichoptere, 2 Coleopteren und 8 Dipteren.

Unter diese Arten können folgende, also 15\%, als Phreathobionten betrachtet werden: Triclade (pigmentfreies Exemplar), Candona eremita Vejd., Asellus (Proasellus) danubialis R. \& M. Codr., Asellus (Proasellus) elegans R. \& M. Codr., Niphargus jovanovici bajusaricus Schell. und Niphargopsis trispinosus Dancau \& Capuşe.

Die übrigen 28 Arten, das heißt 85\%, gehören zur Gruppe der Phreatoxenen.

$\mathrm{Zu}$ bemerken ist, daß Vejdovsky (1882) in den Brunnen von Prag, Jaworovski (1895) in den Brunnen von Krakow und Lwow (Lemberg), Moniez $(1888,1889)$ in den Brunnen im Nordosten Frankreichs und Chappuis (1922) in den Brunnen aus der Umgebung von Basel einen viel geringeren Anteil (zum Beispiel Chappuis $=2 \%$ ) fanden. 


\section{RÉSUMÉ}

Après une introduction, comprenant surtout un historique sommaire des recherches sur la faune des puits, suit une description de la région étudiée où l'on a exploré 13 puits. Ensuite les auteurs exposent leur méthode de travail et indiquent les caractéristiques physico-chimiques de l'eau (température, $\mathrm{pH}$, alcalinité, degré de dureté, contenu en $\mathrm{O}_{2}$, résidu fixe, matières en suspension, $\mathrm{N}_{2} \mathrm{O}_{5}, \mathrm{P}_{2} \mathrm{O}_{5}, \mathrm{NaCl}, \mathrm{Ca}, \mathrm{Fe}$ ).

La faune des puits de Mehadia (v. partie systématique) se compose de 34 espèces: 1 Triclade, 3 Oligochètes, 2 Gastéropodes, 5 Cladocères, 1 Ostracode, 3 Copépodes, 4 Isopodes, 2 Amphipodes, 1 Halacaride, 1 Collembole, des foureaux vides d'un Trichoptère, 2 Coléoptères et 8 Diptères (larves et nymphes).

Parmi ces espèces les suivantes, c'est-à-dire 15\%, peuvent être considérées comme phréatobiontes: Triclade aveugle (non déterminé) ${ }^{4}$ ), Candona eremita Vejd., Asellus (Proasellus) danubialis R. \& M. Codr., Asellus (Proasellus) elegans R. \& M. Codr., Niphargus jovanovici bajuvaricus Schell. et Niphargopsis trispinosus Dancau \& Capuşe.

Le reste des espèces en nombre de 28 , c'est-à-dire $85 \%$, appartiennent au groupe de phréatoxènes.

Il est à remarquer que Vejdovsky (1882) dans les puits de Prague, Jaworowski (1895) dans les puits de Cracovia et de Lwov, Moniez (1888, 1889) dans les puits du Nord-Est de la France et Chappuis (1922) dans ceux des environs de Bâle, ont trouvé une proportion beaucoup plus petite de formes phréatobies ( $p$. ex. Chappuis $=2 \%$ ).

\section{LITERATUR}

Angelier, E. (1953) - Recherches écologiques et biogéographiques sur la faune des sables submergés d'eau douce. Arch. Zool. Epér. et Génér., Paris $90,2,37-162$.

Braun, M. (1881) - Beiträge zur Kenntnis der Fauna Baltica. I. Über Dorpater Brunnenplanarien. Arch. f. d. Naturk. Lis. Esth. und Kurlands 9, 4, Dorpat (Cit. apud Vejdovsky 1882).

Chappuis, P. A. (1920) - Die Fauna der unterirdischen Gewässer der Umgebung von Basel. Arch. f. Hydrobiol., 14, 1-88, Stuttgart, mit 4 Textfig. (Cit. apud Chappuis 1927).

- (1936) - Subterrane Harpaticiden aus Jugoslawien. Bulet. Soc. Stiinte, Cluj. 8, 386-398.

Chilton, Ch. (1893) - The subterranean Crustacea of New-Zeeland, with some general remarks on the Fauna of Caves and Wells. Trans. Linn. Soc. London 6, Ser. II (Cit. apud Spandl: Die Tierwelt der unterirdischen Gewässer - Wien 1926).

Codreanu, R. (1943) - Dendrocelum (sous-genre Dendrocoelides) clujanum n.sp. nouveau Triclade souterrain de Transylvanie. An. Acad. Mem. Sect. Stiin., S. 3, Bucureşti 18, 7, 24.

Dancau, D. und Capuse, I. (1959) - Niphargopsis trispinosus n. sp. - genus new to the Roumanian Fauna (Amphipoda). Folia Balcanica, Skoplje $2,1,8$.

4) Confié a M. le Prof. R. Codreanu qui n'a pu jusqu'à présent l'identifier. 
Dancau, D. (1962) - Niphargus gallicus Schell., Amfipod subteran nou pentru fauna R. P. R. Comunicarile Academiei R. P. R. 13, 2, 123.

Garbini, A. (1896) - Osservazioni biologiche intorno ale aque freatiche veronesi. Verona.

Jaworowski, A. (1895) - Neue Arten der Brunnenfauna von Krakau und Lemberg. Arch.f. Naturgeschichte, Berlin 3, 29-48.

JEANNEL, R. (1937) - Le domaine souterrain. Chap. 5, p. 5. Encyclopédie Française. A. de Monzie, Paris.

Karaman, St. (1935) - Die Fauna der unterirdischen Gewässer Jugoslawiens. Verh. Inter. Ver. theor. angew. Limnologie, Beograd 8, 1, 46-91.

Lundblad, O. (1962) - Die Hydracarinen Schwedens, II. Arkio. f. Zoologi, Ser. $2,14,1$.

Meyer, J. (1782) - Abhandl. von den Würmern der Menschen. Abh. einer Provat-ges. in Böhmen, 5 Prag (Cit. apud H. Spandl 1926).

Moniez, R. (1888-1889) - La faune des eaux souterraines du Département du Nord et en particulier de la ville de Lille. Res. Biol. Nord. France 1.

Мотаş, C (1958) - Freatobiologia, o noua ramura a limnologiei. Natura, Bucureşti 10, 3, 95-105 (La Phréatologie, une nouvelle branche de la Limnologie. Communication présentée à la Session scientifique de la S.S.N.G., Section Zoologie, Bucarest le 18 Octobre 1957).

Motaş, C., Botoşaneanu, L. und Negrea, St. (1962) - Recherches sur la biologie des sources et des eaux phréatiques de la partie centrale de la Plaine Roumaine. Edit. Acad. Rep. Pop. Roum., Bucureşti 1962, 366 pg., 137 fig.

Motaş, G., Dobreanu, E. und Manolache, C. (1948) - Quelques Amphipodes phréaticoles de Roumanie. Bull. Scient. Acad. Roum. 30, 8, 484-492.

Motaş, C. und Orghidan, Tr. (1948) - Quelques Cladocères recuellies dans l'eau phréatique. Not. Bilogicae 6, p. 123-129, fig. 8.

Motaş, C., Tanasachi, J. und Orghidan, Tr. (1958) - Hydrachnelles phréaticoles de la R. P. Roumaine. Acta Soc. Zool. Bohemosl 22, 4, 293-333.

Pratz, E. (1866) - Über einige im Grundwasser lebende Tiere, Petersburg (Cit. apud Chappuis 1927).

Rolland, G. (1894) - Les animaux rejetés vivants par les puits jaillissants de L'Oued Rir. Rev. Scient. October 1894, Paris (Cit. apud A. Viré : Essai sur la faune obscuricole de France, étude particulière de quelques faunes zoologiques. 1899, p. 145. J. B. Baillère \& Tils, Paris.)

Schrank, F. v. P. - Prilog poznavanju faune aladkovodnih Korepnjaka hrvatske. Rad. jugoslovenske akademije znanosti $i$ umjrthesti, Agram. 92 (Cit. apud H. Spandl 1926, p. 44).

Tanasaghi, J. und Orghidan, Tr. (1955) - Hidracarieni orbi din apele freatice. Bul. Stiinn Sect. Stiin. Biol. Agron. Geol.Geogr. 7, 2, p. 369381.

VEJD OVSKY, Fr. (1882) - Tierische Organismen in den Brunnenwässern von Prag, p. 1-66, Pl. 8. Selbstverlag - In Commission Franz Rivnac, Prag.

Zveткоv, L. (1964) - Sur l'identification de l'Ingolfiella petkovskii Karam. (Crustacea - Isopoda). Acad. bulg. Scieences, Bull. de l'Institut et Musée de Zool., Sofia, 15, p. 185-190, 14. 


\section{Anmerkung}

Bevor das Manuskript dieser Arbeit sowie ein Nekrolog für Dr. Karl Viets an das International Journal of Speleology gelangte, umkreisten sie ganz Jugoslawien, von Skopje nach Belgrad und alsdann nach Zagreb. Das odysseische Abenteuer der beiden Arbeiten, ist gewiß von Interesse, doch ist dies nicht die Gelegenheit, es zu erzählen.

Eine lange Zeit als verloren betrachtet, wurde die Kopie des Manuskriptes dieser Arbeit von Prof. Dr. R. Husson von der Wissenschaftlichen Fakultät aus Dijon im Jahre 1964 an den Verlag J. Cramer (Weinheim) gesendet.

Während der Text gesetzt wurde, erhielten wir zu unserem großen Erstaunen im September dieses Jahres Auszüge, die schon in Zagreb publiziert wurden, in Bioloski Glasnik (15. 4. 1962).

Unter der Bedingung, daß einige Zusätze im Text und in der Bibliographie vorgenommen werden, gestatten wir, da $\beta$ die Arbeit nochmals abgedruckt wird. 